\title{
ALMOST EVERY QUASINILPOTENT HILBERT SPACE OPERATOR IS A UNIVERSAL QUASINILPOTENT'1
}

\author{
DOMINGO A. HERRERO
}

\begin{abstract}
Let $Q$ be a quasinilpotent operator acting on a complex separable infinite dimensional Hilbert space; then either $Q^{k}$ is compact for some positive integer $k$, or the closure of the similarity orbit of $Q$ contains every quasinilpotent operator. Analogous results are shown to be true for the Calkin algebra and for nonseparable Hilbert spaces. For the nonseparable case, the analogous result is true for the closed bilateral ideal $g$, strictly larger than the ideal of compact operators, if and only if $g$ is not the ideal associated with an $\boldsymbol{\aleph}_{0}$-regular limit cardinal. For the ideal of compact operators, the problem remains open.
\end{abstract}

1. Introduction. Let $\mathcal{L}(\mathcal{H C})$ be the algebra of all (bounded linear) operators acting on the complex separable infinite dimensional Hilbert space $\mathcal{H}$. A quasinilpotent operator $Q$ is called a universal quasinilpotent operator (u.q.o.) if the closure $\delta(Q)^{-}$of its similarity orbit

$$
\delta(Q)=\left\{W Q W^{-1}: W \text { is invertible in } \mathcal{L}(\mathcal{H})\right\}
$$

contains the set $\mathbf{Q}(\mathcal{H})$ of all quasinilpotent operators.

The existence of a u.q.o. was proven in [7] and a strong refinement of this result was obtained in $[1, \S 7]$. The main result of this note provides a complete characterization of the set of all universal quasinilpotent operators.

THEOREM 1. Let $\mathcal{H C}$ be a complex separable infinite dimensional Hilbert space and let $\mathrm{N}(\mathcal{H C})$ and $\mathcal{H}(\mathcal{H})$ denote the set of all nilpotent operators and the ideal of compact operators, respectively. Then $Q \in \mathcal{L}(\mathcal{H})$ is a universal quasinilpotent operator if and only if it belongs to $\mathbf{Q}_{a e}(\mathcal{H})=\mathbf{Q}(\mathcal{H}) \backslash[\mathbf{N}(\mathcal{H})+\mathcal{H}(\mathcal{H})]$.

Furthermore, $\mathbf{Q}_{a e}(\mathcal{H})$ is a $G_{\delta}$ dense subset of $\mathbf{Q}(\mathcal{H})$.

Let $\pi$ be the canonical projection of $\mathscr{L}(\mathcal{H})$ onto the Calkin algebra $\mathscr{Q}(\mathcal{H C})=\mathscr{L}(\mathcal{H}) / \mathscr{K}(\mathcal{H})$. It is completely apparent that a quasinilpotent $T$ belongs to $\mathbf{N}(\mathcal{H})+\mathscr{K}(\mathcal{H C})$ if and only if $\pi(T)$ is a nilpotent in $\mathscr{Q}(\mathcal{H})$ [10],

Received by the editors July 18, 1977 and, in revised form, January 24, 1978.

AMS (MOS) subject classifications (1970). Primary 41A65, 47A65, 47B05; Secondary 46L05, 47A55.

Key words and phrases. Nilpotent operator, quasinilpotent operator, similarity orbit, universal quasinilpotent operator, compact operator, Calkin algebra, Voiculescu's noncommutative version of Weyl-von Neumann theorem, representation of $C^{*}$-algebras, quasitriangular operator, nonseparable Hilbert space, $\boldsymbol{N}_{0}$-irregular cardinal.

${ }^{1}$ This work has been partially supported by CONICIT (Venezuela), Grant No. 51.26.S1-0894. 
[13], and therefore every u.q.o. is an element of $\mathbf{Q}_{a e}(\mathcal{F C})$ [1], [8, Proposition 1 (vii)]. On the other hand, we have: (a) $\mathbf{Q}(\mathcal{H C})$ is a $G_{\delta}$ subset of $\mathcal{L}(\mathcal{H C})$ [5, proof of Theorem (3.2)]; (b) $\pi[\mathbf{N}(\mathcal{H})+\mathcal{K}(\mathcal{H C})]$ and $\mathbf{N}(\mathcal{H})+\mathcal{K}(\mathcal{H})$ are $F_{\sigma}$ subsets of $\mathcal{Q}(\mathcal{H C})$ and $\mathcal{L}(\mathcal{H C})$, respectively [6, Corollary 3]; (c) $\mathbf{Q}_{a e}(\mathcal{H C})$ is a dense subset of $\mathbf{Q}(\mathcal{H})$ [7]; and therefore, (d) $\mathbf{Q}_{a e}(\mathcal{F C})$ is a $G_{\delta}$ dense subset of $\mathbf{Q}(\mathcal{F})$, i.e., contains "almost every" quasinilpotent.

Thus, in order to complete the proof of Theorem 1 it remains to show that every element of $\mathbf{Q}_{a e}(\mathcal{H C})$ is actually a u.q.o.; this will be done in $\$ 2$ below, which also contains the analogous result for the Calkin algebra $\mathcal{Q}(\mathcal{H})$. $\$ 3$ contains the natural extensions of Theorem 1 to the algebra of operators and the closed bilateral ideals which are strictly larger than $\mathcal{K}(\mathcal{H})$, for nonseparable Hilbert spaces. The analogous result for $\mathscr{K}(\mathcal{H})$ (Conjecture 1 of [7]) remains an open problem.

2. Characterization of the set of u.q.o. Let $Q \in \mathbf{Q}_{a e}(\mathcal{F C})$ and let $\rho$ be a faithful representation of the $C^{*}$-algebra $\left[C^{*}(Q)+\mathscr{K}(\mathcal{H})\right] / \mathcal{K}(\mathcal{H})$, in the separable Hilbert space $\mathcal{H}_{\rho}$, where $C^{*}(Q)$ denotes the $C^{*}$-algebra generated by $Q$. Clearly, $Q_{1}=\rho \circ \pi(Q) \in \mathbf{Q}\left(\mathcal{H}_{\rho}\right) \backslash \mathbf{N}\left(\mathcal{H}_{\rho}\right)$ and, by Voiculescu's theorem [14, Theorem 1.3], the closure $Q(Q)^{-}$of the unitary orbit of $Q$ contains an operator $Q_{2} \approx Q \oplus Q_{1}^{(\infty)}$, where $\approx, \oplus$ and $T^{(\alpha)}$ denote "unitarily equivalent to", "orthogonal direct sum" and "orthogonal direct sum of $\alpha(0 \leqslant \alpha \leqslant \infty)$ copies of $T$ ", respectively.

Since $Q$ and $Q_{1}$ are quasinilpotent operators, they are quasitriangular [2] and therefore there exist compact operators $K_{0}, K_{1}, K_{2}, \ldots$, such that $\left\|K_{n}\right\|<2^{-n}, \quad T_{0}=Q-K_{0}=\left(t_{i j}^{0}\right)_{i, j=1}^{\infty}$ and $T_{n}=Q^{1}-K_{n}=\left(t_{i j}^{n}\right)_{i, j=1}^{\infty}, \quad n=$ $1,2, \ldots$, where $\left(t_{i j}^{n}\right)_{i, j=1}^{\infty}$ is an upper triangular matrix with respect to a suitable ONB $\left\{e_{j}^{n}\right\}_{j=1}^{\infty}$ for all $n=0,1,2, \ldots$ Furthermore, by using the semicontinuity of the spectrum, $K_{n}$ can be chosen so that $t_{j j}^{n}=0$ for all $j=1,2, \ldots$, and for all $n=0,1,2, \ldots$.

Let $T=\bigoplus_{n=0}^{\infty} T_{n}$; then $T=\left(T_{i j}\right)_{i, j=1}^{\infty}$ with respect to the orthogonal direct sum $\mathcal{H}_{\infty}=\bigoplus_{l=1}^{\infty} \mathcal{K}_{l}$, where $\mathcal{H}_{l}$ is the closed linear span of the orthonormal system $\left\{e_{l}^{n}\right\}_{n=0}^{\infty}(l=1,2, \ldots), T_{i j}=0$ if $i \geqslant j$ and $T_{i j}$ is a diagonal (normal) operator with diagonal entries $t_{i j}^{0}, t_{i j}^{1}, t_{i j}^{2}, \ldots$, if $i<j$. Clearly, $K=\bigoplus_{n=0}^{\infty} K_{n}$ $\in \mathcal{K}\left(\mathcal{H}_{\infty}\right)$ and $Q_{2}=T+K$.

Now we are in a position to apply the same argument as in the first part of the proof of Theorem 1.2 in [4]. Let $P_{m}$ be the orthogonal onto $\bigoplus_{l=1}^{m} \mathcal{H}_{l}$ $(m=1,2, \ldots)$ and let $\tau$ be a faithful representation of the $C^{*}$-algebra $\left[C^{*}\left(T ; P_{1}, P_{2}, \ldots\right)+\mathcal{K}\left(\mathcal{H}_{\infty}\right)\right] / \mathcal{K}\left(\mathcal{H}_{\infty}\right)$ in the separable Hilbert space $\mathcal{H}_{\tau}$, where $C^{*}\left(T ; P_{1}, P_{2}, \ldots\right)$ is the separable $C^{*}$-algebra generated by $T$ and the projections $P_{m}, m=1,2, \ldots$.

Let $S=\tau \circ \pi(T)$ and $R_{m}=\tau \circ \pi\left(P_{m}\right), m=1,2, \ldots$ Since $P_{m} T P_{m}=$ $P_{m} T$, it readily follows that $R_{m} S R_{m}=R_{m} S$ and therefore $R_{m} S R_{m}$ is the direct sum of an $m \times m$ upper triangular operator matrix with the 0 operator; furthermore, the upper triangular matrix has 0's in the diagonal entries; hence, $\left(R_{m} S R_{m}\right)^{m}=0$ for all $m=1,2, \ldots$ On the other hand, since $\tau$ is faithful 
and $\pi(T)^{k-1}=\pi\left(Q_{2}\right)^{k-1} \neq 0$ for all $k=1,2, \ldots$, given $k$ there exists a minimal index $m(k)$ such that $\left(R_{m(k)} S R_{m(k)}\right)^{k-1} \neq 0$. Then by [14, Theorem 1.3], there exists $Q_{3} \approx Q \oplus S^{(\infty)}$ in $\mathcal{Q}(Q)^{-}$and, by Rota's corollary [11] and standard arguments (see, e.g., [1], [8]), $0^{(\infty)} \oplus\left(R_{m(k)} S R_{m(k)}\right)^{(\infty)} \in \mathcal{S}(Q)^{-}$for all $k=1,2, \ldots$.

Let $q_{k} \in \mathfrak{L}\left(\mathbf{C}^{k}\right)$ be the truncated shift of order $k$ (i.e., $q_{k} e_{1}=0, q_{k} e_{j}=e_{j-1}$ for all $j=2,3, \ldots, k$, with respect to the canonical ONB of $\left.\mathbf{C}^{k}\right)$. Since $\operatorname{Ran}\left[\left(R_{m(k)} S R_{m(k)}\right)^{(\infty)}\right]^{k-1}$ contains an infinite dimensional (closed) subspace, it follows from [1, Lemma 2.1] that $q_{k}^{(\infty)} \oplus 0^{(\infty)} \in \delta(Q)^{-}$for all $k=$ $1,2, \ldots$, and therefore, by [1, Theorem 7.1], $Q$ is a u.q.o.

Recall that the similarity orbit of an element of $\mathscr{Q}(\mathcal{H C})$ is equal to $\delta(a)=$ $\{\tau(a): \tau \in$ Automorphisms of $\mathscr{Q}(\mathcal{H})\}=\left\{w a w^{-1}: w\right.$ is invertible in $\left.\mathscr{Q}\right\}$ [1], [12] and that every nilpotent (quasinilpotent, resp.) in $\mathscr{Q}(\mathcal{H C})$ can be lifted to a nilpotent of the same order (quasinilpotent, resp.) in $\mathcal{L}(\mathcal{H})$ [10], [13]. By using these observations and Theorem 1, we can easily obtain the following nice

Corollary 1. Let $a \in \mathbb{Q}(\mathcal{H})$; then the following are equivalent:

(i) $\delta(a)^{-}$contains every quasinilpotent element of $\mathbb{Q}(\mathcal{H})$;

(ii) $\delta(a)^{-}$contains every nilpotent element of $\mathscr{Q}(\mathcal{H C})$;

(iii) $\delta(a)^{-}$contains a sequence $\left\{n_{j(k)}\right\}_{k=1}^{\infty}$ of nilpotent elements of $\mathbb{Q}(\mathcal{H})$ such that $\lim (k \rightarrow \infty) j(k)=\infty$ and $n_{j(k)}^{j(k)} \neq 0$;

(iv) $a$ is quasinilpotent, but not nilpotent.

Proof. (i) $\Rightarrow$ (ii) $\Rightarrow$ (iii) $\Rightarrow$ (iv). The first two are trivial implications and the third one follows from [8, Proposition 1(i) and (vii)].

(iv) $\Rightarrow$ (i) If $a$ satisfies (iv); then it can be lifted to an $A \in \mathbf{Q}_{a e}(\mathcal{H})$ [10], [13]. Since, by Theorem $1, \delta(\mathscr{Q})^{-}$contains every quasinilpotent of $\mathcal{L}(\mathcal{H})$, it follows from [10], [13] that $\delta(a)^{-}=\delta[\pi(\mathscr{Q})]^{-} \supset \pi[\delta(\mathscr{Q})]^{-} \supset\{q: q$ is a quasinilpotent of $\mathscr{Q}(\mathcal{H})\}$.

3. The nonseparable case. Throughout this section, $\mathcal{H}$ will denote a nonseparable complex Hilbert space of (topological) dimension $h>\boldsymbol{\aleph}_{0}$. Given a cardinal number $\alpha, \kappa_{0} \leqslant \alpha \leqslant h, g_{\alpha}=\left\{T \in \mathcal{L}(\mathcal{F})\right.$ : $\operatorname{dim}(\operatorname{Ran} T)^{-}<$ $\alpha\}$ is a bilateral ideal of $\mathcal{L}(\mathcal{H C})$ and $g_{\alpha}=g_{\alpha}^{-}$is a closed bilateral ideal (we shall simply say " $\mathscr{g}_{\alpha}$ is an ideal"). Furthermore, every ideal of $\mathcal{L}(\mathcal{H C})$ is either trivial or equal to $g_{\alpha}$ for some $\alpha, \aleph_{0} \leqslant \alpha \leqslant h$ [3].

A quasinilpotent $Q \in g_{\alpha}$ is a u.q.o. for the ideal $g_{\alpha}$ if $\delta(Q)^{-}$contains every quasinilpotent element of $g_{\alpha}$. It is clear that if $Q$ satisfies the above conditions, then $Q^{k}$ cannot belong to any strictly smaller ideal $(k=$ $1,2, \ldots)$. The existence of some u.q.o. for the ideal $g_{\alpha}$ strongly depends on the properties of $\alpha$ (see [7]). The best possible result, except perhaps for the ideal $\mathscr{K}$ of compact operators, is contained in Theorem 2 below. Recall that a cardinal number $\alpha$ is $\kappa_{0}$-irregular if $\alpha=\sup _{n} \alpha_{n}$ for a strictly increasing sequence $\left\{\alpha_{n}\right\}_{n=1}^{\infty}$ of cardinals [3]; then, we have

TheOREM 2. (i) $Q \in \mathcal{L}(\mathcal{H})$ is a u.q.o. if and only if $Q$ is quasinilpotent and $Q^{k} \notin g_{h}$, the maximal ideal, for any $k=1,2, \ldots$ 
(ii) If either $\alpha=\aleph_{\nu+1}$, for some ordinal number $\nu$, or $\alpha$ is $\aleph_{0}$-irregular and strictly larger than $\kappa_{0}$, then $Q \in \mathcal{g}_{\alpha}$ is a u.q.o. for $\mathcal{g}_{\alpha}$ if and only if $Q$ is quasinilpotent and $Q^{k} \notin \mathcal{F}_{\beta}$ for any $\beta<\alpha$ and for any $k=1,2, \ldots$

(iii) If $\alpha$ is $\aleph_{0}$-regular, but not of the form $\aleph_{\nu+1}$ for some ordinal number $\nu$, then $g_{\alpha}$ does not have a u.q.o.

Proof. (ii) Let $Q \in g_{\alpha}$, where $\alpha>\aleph_{0}$ is an $\aleph_{0}$-irregular cardinal ( $\alpha=$ $\aleph_{\nu+1}$ for some ordinal number $\nu$, resp.), be a quasinilpotent operator such that no power of $Q$ belongs to a strictly smaller ideal. Then $Q$ admits a decomposition. $Q=0^{\left(h^{\prime}\right)} \oplus\left\{\oplus Q_{\gamma}: \gamma \in \Gamma\right\}$, where $Q_{\gamma}$ is a quasinilpotent operator acting on a separable infinite dimensional Hilbert space $\mathcal{H}_{\gamma}, Q_{\gamma}^{k-1} \notin \mathcal{K}\left(\mathcal{F}_{\gamma}\right)$ $(k=1,2, \ldots), \mathbf{C}(\Gamma)=\alpha\left(\mathbf{C}(\Gamma)=\aleph_{v}\right.$, resp. $)$ and $h^{\prime}=h$ or 0 , according to $\alpha<h$ or $\alpha=h$, resp. ( $h^{\prime}=h$, resp.). Furthermore, given $k$, the cardinals of the subsets $\Gamma_{k, n}=\left\{\gamma \in \Gamma:\left\|Q_{\gamma}^{k-1} x\right\| \geqslant(1 / n)\|x\|\right.$ for all $x$ in a suitable infinite dimensional subspace of $\left.\mathcal{H}_{\gamma}\right\}$ satisfy $\mathbf{C}\left(\Gamma_{k, n}\right)<\alpha=\sup _{n} \mathbf{C}\left(\Gamma_{k, n}\right)$ (there exists $\delta_{k}>0$ such that $\left\|Q_{\gamma}^{k-1} x\right\| \geqslant \delta_{k}\|x\|$ for all $x$ in a suitable infinite dimensional subspace of $\mathcal{H}_{\gamma}$, for all $\gamma \in \Gamma$, resp.). The details of the decomposition can be easily checked by using the results of [3], [9].

Thus, given $\beta<\alpha$, there exists an $m$ such that $\mathbf{C}\left(\Gamma_{k, m}\right) \geqslant \beta$. Applying the arguments of the proof of Theorem 1 to $Q_{\gamma}$ for all $\gamma \in \Gamma_{k, m}$ (for all $\gamma \in \Gamma$, resp.), we conclude that $q_{k}^{(\beta)} \oplus 0^{(h)} \in \delta(Q)^{-}$for all $\beta<\alpha$ and for all $k=1,2, \ldots$, and therefore $Q$ is a u.q.o. for $g_{\alpha}[1]$.

Conversely, if $Q$ is a u.q.o. for $g_{\alpha}$, then it is clear that if $Q^{k} \in g_{\beta}$ for some $k$ and some $\beta<\alpha$, then $\delta(Q)^{-} \subset\left\{A \in \mathcal{L}(\mathcal{H}): A^{k} \in \mathcal{G}_{\beta}\right\}$, a contradiction [1], [8].

(i) follows by a minor modification of the above proof for the case when $\alpha=\aleph_{\nu+1}$. Finally, (iii) is contained in [7, Theorem 3].

\section{REFERENCES}

1. J. Barría and D. A. Herrero, Closure of similarity orbits of nilpotent operators. II (to appear).

2. R. G. Douglas and C. Pearcy, A note on quasitriangular operators, Duke Math. J. 37 (1970), 177-188.

3. G. Edgar, J. Ernest and S. G. Lee, Weighing operator spectra, Indiana Univ. Math. J. 21 (1971), 61-80.

4. C. Foias, C. Pearcy and D. Voiculescu, Biquasitriangular operators and quasisimilarity (to appear).

5. S. Grabiner, Nilpotents in Banach algebras, J. London Math. Soc. (2) 14 (1976), 7-12.

6. D. A. Herrero, Normal limits of nilpotent operators, Indiana Univ. Math. J. 23 (1974), 1097-1108.

7. __ Universal quasinilpotent operators, Acta Sci. Math. (Szeged) 38 (1976), 291-300.

8. Clausura de las órbitas de similaridad de operadores en espacios de Hilbert, Rev. Un. Mat. Argentina 27 (1976), 244-260.

9. Norm limits of nilpotent operators and weighted spectra in non-separable Hilbert space, Rev. Un. Mat. Argentina 27 (1975), 83-105.

10. C. L. Olsen, A structure theorem for polynomially compact operators, Amer. J. Math. 93 (1971), 686-698.

11. G.-C. Rota, On models for linear operators, Comm. Pure Appl. Math. 13 (1960), 469-472.

12. S. Sakai, $C^{*}$-algebras and $W^{*}$-algebras, Ergebnisse der Math. und ihrer Grenzgebiete, Bd. 60, Springer-Verlag, New York and Berlin, 1971. 
13. J. G. Stampfli, Compact perturbations, normal eigenvalues and a problem of Salinas, J. London Math. Soc. (2) 9 (1974), 165-175.

14. D. Voiculescu, A non-commutative Weyl-von Neumann theorem, Rev. Roumaine Math. Pures Appl. 21 (1976), 97-113.

Departamento de Matemáticas, instituto de Investigaciones Científicas, Apartado Postal $N^{\circ} 1827$, Caracas 101, Venezuela 